\title{
Actinobacillus ureae
}

National Cancer Institute

\section{Source}

National Cancer Institute. Actinobacillus ureae. NCI Thesaurus. Code C86103.

A species of anaerobic, Gram negative, rod shaped bacteria assigned to the phylum Proteobacteria. This bacteria is oxidase and catalase positive, reduces nitrate, hydrolyzes urea and is indole negative. A. ureae is found rarely as a commensal organism of the human respiratory tract and may cause respiratory tract infections in humans. 